\title{
Effect of 1,25-dihydroxycholecalciferol on glucose metabolism in gestational diabetes mellitus
}

\author{
P.M . R udnicki, L . M ølsted-Pedersen \\ D iabetes Center, D epartment of O bstetrics and G ynecology, R igshospitalet, U niversity of Copenhagen, D enmark
}

Summary The effect of 1,25-dihydroxyvitamin D supplement on glucose metabolism was evaluated in 12 women with gestational diabetes mellitus (GDM). All women had an oral glucose tolerance test (OGTT) performed at inclusion in the study. Thereafter, each patient was instructed to continue their normal diet during the following 2 days, after which they received $2 \mu \mathrm{g} / \mathrm{m}^{2} \mathrm{E}$ talpha i. v. $2 \mathrm{~h}$ prior to the second OGTT. D uring the next 14 days each patient received $0.25 \mu \mathrm{g}$ Etalpha orally, after which a third OGTT was performed. On average the level of 1,25dihydroxyvitamin $\mathrm{D}_{3}$ at inclusion at baseline significantly increased after i.v. 1,25-dihydroxyvitamin $D_{3}$ (from 92 to $138 \mathrm{ng} / \mathrm{l}[\mathrm{p}<0.001]$ ), but returned to the baseline level after 2 weeks of oral E talpha ( $85 \mathrm{ng} / \mathrm{l}$ ). Simultaneously, the glucose level decreased from 5.6 to $4.8 \mathrm{mmol} / \mathrm{l}(p<0.01)$ after i. v. treatment with 1,25-dihydroxyvitamin $D_{3}$, but did not differ significantly from inclusion following 2 weeks of oral Etalpha. There was no difference between the glucose concentrations during OGTT prior to and after i.v. or oral 1,25 -dihydroxyvitamin $\mathrm{D}_{3}$, in contrast to the insulin levels which tended to be lower after both i.v. and oral supplementation. In a multiple regression model including 1,25-dihydroxyvitamin $\mathrm{D}_{3}$, insulin , weight and height against glucose, only 1,25dihydroxyvitamin $D_{3}$ and insulin were selected for the final model $\left(r^{2}=0.73, p<0.0001\right)$. O ur results suggest that supplements of 1,25-dihydroxyvitamin $\mathrm{D}_{3}$ influence glucose metabolism in patients with GDM probably by increasing the insulin sensitivity. [D iabetologia (1997) 40: 40-44]

Keywords Gestational diabetes mellitus, cholecalciferols.
Several studies have demonstrated specific receptors for 1,25-dihydroxyvitamin $D$ in pancreatic beta cells suggesting a role for 1,25 -dihydroxyvitamin $D$ in the regulation of insulin secretion $[1,2]$. O thers have shown that vitamin D depleted animals exhibited impaired glucose clearance and insulin secretion, which were abolished by vitamin D supplement [3-6]. This beneficial effect appeared early after i. v. injection of 1,25-dihydroxyvitamin D.

R eceived: 9 J uly 1996 and in revised form: 12 September 1996

Corresponding author: Dr. P.M. Rudnicki, Diabetes Center, Department of Obstetrics and Gynecology, R igshospitalet, U niversity of Copenhagen, DK -2100 Copenhagen, D enmark Abbreviations: GDM, Gestational diabetes mellitus; NEFA, non-esterified fatty acids; OGTT, oral glucose tolerance test.
In humans the number of studies evaluating the effect of vitamin $D$ on glucose metabolism are increasing. R ecently, studies have shown that vitamin $D$ depleted humans have reduced insulin secretion [7, 8], but this was reversed after vitamin $D$ treatment for 3-6 months. In diabetic patients bone disease is often observed suggesting that this group also suffers from impaired vitamin D metabolism. So far, however, conflicting results have been reported on the level of 1,25-dihydroxyvitamin $D$ in diabetic patients $[9,10]$. This probably explains the diverging results of vitamin $D$ treatment on glucose tolerance in this group $[11,12]$.

Others have evaluated the effect of vitamin D treatment in renal failure $[13,14]$. I n end-stage renal failure the circulating level of 1,25-dihydroxyvitamin D decreases and at the same time impaired glucose 
tolerance arises [15]. M ak [13] demonstrated that i. v. administration of $2 \mu \mathrm{g} / \mathrm{m}^{2}$ of 1,25-dihydroxyvitamin $D$ reversed glucose intolerance in such patients. Similarly, Quesada et al. [14] showed that 2 weeks of treatment with oral calcitriol $0.5 \mu \mathrm{g} /$ day significantly increased serum insulin concentration.

The presence of insulin resistance in late human pregnancy is well-known [16]. D uring this period the production of 1,25-dihydroxyvitamin $D$ increases [17]. In gestational diabetes mellitus (G D M ) and especially in insulin-dependent diabetic pregnant women the concentration of 1,25-dihydroxyvitamin D remains low in late pregnancy compared to normal pregnant women [18, 19].

It is not known whether 1,25-dihydroxyvitamin D increases insulin secretion in G D M . We therefore undertook a study in order to evaluate whether 1,25-dihydroxyvitamin $D_{3}$ supplement could improve glucose metabolism in such patients.

\section{Subjects, materials and methods}

Twenty consecutive women admitted to hospital because of suspicion of GDM were invited to participate in the study. Of these, $12 \mathrm{had}$ an abnormal glucose tolerance test defined as two or more serum glucose values 3 SD above normal average and without abnormal glucose tolerance or glucosuria after pregnancy. The gestational age at inclusion (assessed by ultrasound measurements of the biparietal diameter in week 16 of pregnancy) was on average 27 weeks (range 17-33) and height and weight were $165 \mathrm{~cm}(163-8 \mathrm{~cm})$ and $80 \mathrm{~kg}(53-102 \mathrm{~kg})$, respectively. A II were nulliparous and all delivered healthy children appropriate for gestational age. N one of the patients received insulin preparations, supplements of vitamin D or calcium during pregnancy. W ritten informed consent was obtained from all subjects. The study was approved by the local ethical committee.

A $n$ oral glucose tolerance test (OGTT) was performed in the morning at the same time for each group after an overnight fast using an oral dose of $75 \mathrm{~g}$ glucose. B lood samples were taken at baseline $(-5 \mathrm{~min})$ and after 30,60,90, 120, 150 and $180 \mathrm{~min}$ for measurements of glucose and insulin. Concentrations of triglycerides, total cholesterol, non-esterified fatty acids (NE FA), parathyroid hormone, 1,25-dihydroxyvitamin $D_{3}$, ionized calcium adjusted to $\mathrm{pH}$ 7.40, magnesium and phosphate were measured only at baseline, $-5 \mathrm{~min}$. E ach patient was instructed to continue their normal diet during the following 2 days, after which they underwent a second O G TT after an overnight fast. Two hours prior to this O GTT the patients received $2 \mu \mathrm{g} / \mathrm{m}^{2} \mathrm{E}$ talpha i.v. D uring the following 14 days each patient received daily $0.25 \mu \mathrm{g} \mathrm{E}$ talpha orally after which a third OGTT was performed following an overnight fast. The final drug dose was taken the day the O G TT was performed. D uring the latter period the patients received a restricted diet until 3 days prior to the third OG TT. The patients initially received 20 tablets and had to return any remaining tablets after the 2week treatment period.

M ethods. Insulin was measured by an enzyme linked immonosorbent assay (E LISA) as described previously [20]. The intraand interassay CV s were 6.4 and $5.0 \%$, respectively. D etection limit was $3 \mathrm{pmol} / \mathrm{l}$.
Table 1. B aseline fasting levels of measured variables in gestational diabetic women prior to, and after intravenous and oral treatment with 1,25-dihydroxyvitamin D

\begin{tabular}{|c|c|c|c|}
\hline $\begin{array}{l}\text { Serum/plasma } \\
\text { parameter }\end{array}$ & Inclusion & $\begin{array}{l}2 \mathrm{~h} \text { after } \mathrm{i} . \mathrm{v} . \\
\text { vitamin } \mathrm{D}\end{array}$ & $\begin{array}{l}14 \text { days after } \\
\text { oral vitamin D }\end{array}$ \\
\hline Glucose (mmol/l) & $5.6 \pm 0.8$ & $4.8 \pm 0.6^{a}$ & $5.3 \pm 0.8$ \\
\hline Insulin (pmol/l) & $70 \pm 34$ & $65 \pm 24$ & $62 \pm 22$ \\
\hline $\begin{array}{l}\text { Triglycerides } \\
(\mathrm{mmol} / \mathrm{l})\end{array}$ & $2.96 \pm 1.12$ & $2.85 \pm 1.04$ & $2.69 \pm 1.20$ \\
\hline Cholesterol $(\mathrm{mmol} / \mathrm{l})$ & $6.3 \pm 1.0$ & $6.2 \pm 1.1$ & $6.1 \pm 1.4$ \\
\hline NEFA $(\mathrm{mmol} / \mathrm{l})$ & $0.92 \pm 0.34$ & $0.97 \pm 0.43$ & $0.76 \pm 0.46$ \\
\hline $\begin{array}{l}\text { 1,25-dihydroxy- } \\
\text { vitamin } D_{3}(\mathrm{ng} / \mathrm{l})\end{array}$ & $92 \pm 27$ & $138 \pm 27^{b}$ & $85 \pm 19$ \\
\hline $\begin{array}{l}\text { Parathyroid } \\
\text { hormone (ng/l) }\end{array}$ & $35 \pm 14$ & $37 \pm 16$ & $35 \pm 14$ \\
\hline $\begin{array}{l}\text { Ionized calcium } \\
(\mathrm{mmol} / \mathrm{l})\end{array}$ & $1.24 \pm 0.06$ & $1.26 \pm 0.06$ & $1.25 \pm 0.05$ \\
\hline M agnesium (mmol/l) & $0.74 \pm 0.05$ & $0.74 \pm 0.03$ & $0.72 \pm 0.07$ \\
\hline Phosphate $(\mathrm{mmol} / \mathrm{l})$ & $1.09 \pm 0.12$ & $1.07 \pm 0.13$ & $1.06 \pm 0.08$ \\
\hline
\end{tabular}

Values are mean \pm SD

${ }^{a} p<0.01 ;{ }^{b} p<0.001$

The active form of vitamin $D\left(1,25(\mathrm{OH})_{2} \mathrm{D}_{3}\right)$ was measured by radioimmunoassay [21]. 1,25-dihydroxyvitamin $D_{3}$ was extracted by incubation with a highly specific solid phase monoclonal anti-1,25D and reconstituted eluates were incubated overnight with a highly specific 1,25D sheep anti-1,25D to which ${ }^{125}-1,25 \mathrm{D}$ is added. The intra- and interassay CV s were 7 and $14 \%$, respectively; the detection limit of the assay was 3 $\mathrm{ng} / \mathrm{l}$.

I ntact parathyroid hormone was measured by an immunoradiometric assay [22]. The intra- and interassay CV s were 5 and $6 \%$, respectively. The detection limit of the assay was $3 \mathrm{ng} / \mathrm{l}$.

Plasma glucose, triglycerides, total cholesterol and NEFA were measured using enzymatic colorimetric tests. I onized calcium was measured at pH 7.40 using an ICA 2 (Radiometer, Denmark). Serum concentrations of phosphate were measured by a multichannel analyser (Technicon SM A C 3 A naIyzer; Terrytown, N.Y., U SA ). Serum magnesium was measured by atomic absorption spectrophotometry.

\section{Statistical analysis}

A II data are presented as mean \pm SD. A nalysis of variance was used for evaluation of time-related changes. Wilcoxon signed rank test was used for comparison of groups. Correlations were calculated by linear regression analysis by the least square method (Statgraphics Inc., version 2.6). A p-value less than 0.05 was considered statistically significant.

\section{Results}

From Table 1 it appears that the serum level of 1,25dihydroxyvitamin $D_{3}$ at inclusion significantly increased after i.v. 1,25-dihydroxyvitamin $D_{3}$ from 92 to $138 \mathrm{ng} / \mathrm{l}(\mathrm{p}<0.001)$, but did not differ significantly from the inclusion level after oral supplement of 1,25-dihydroxyvitamin $D_{3}$ (85 ng/l). Simultaneously, 


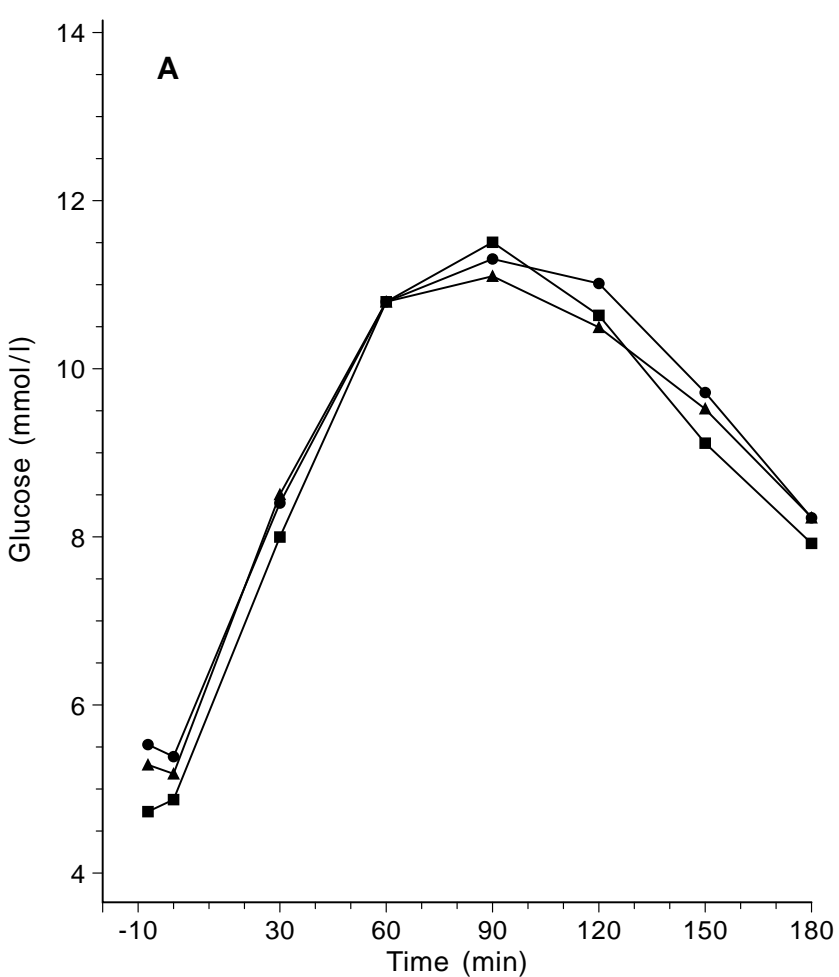

Fig. 1A , B. Plasma glucose (A) and serum insulin (B) levels in response to oral glucose tolerance test $(75 \mathrm{~g})$ in pregnant women with gestational diabetes mellitus without supplementation of 1,25-dihydroxyvitamin (•), and after intravenous injection ( $\mathbf{\square})$ and oral treatment with 1,25-dihydroxyvitamin D ( $\mathbf{\Delta})$

Table 2. Changes in insulin concentrations during OGTT in percent of baseline values

\begin{tabular}{llll}
\hline $\begin{array}{l}\text { Time } \\
\text { (min) }\end{array}$ & Inclusion & $\begin{array}{l}\text { 2 h after i.v. } \\
\text { vitamin D }\end{array}$ & $\begin{array}{l}\text { 14 days after } \\
\text { oral vitamin D }\end{array}$ \\
\hline Baseline $=0$ & 100 & 100 & 100 \\
30 & $355 \pm 165$ & $333 \pm 127$ & $353 \pm 141$ \\
60 & $572 \pm 208$ & $479 \pm 221$ & $483 \pm 150$ \\
90 & $779 \pm 209$ & $625 \pm 216$ & $657 \pm 232$ \\
120 & $926 \pm 306$ & $723 \pm 292$ & $780 \pm 346$ \\
150 & $743 \pm 209$ & $640 \pm 157$ & $717 \pm 367$ \\
180 & $587 \pm 231$ & $451 \pm 166$ & $542 \pm 264$ \\
\hline
\end{tabular}

Values are mean \pm SD

the level of glucose decreased from 5.6 to $4.8 \mathrm{mmol} / \mathrm{l}$ $(p<0.01)$ after i.v. treatment with 1,25-dihydroxyvitamin $D_{3}$, but did not differ significantly from inclusion level after 2 weeks of oral 1,25-dihydroxyvitamin $D_{3}$. N o other parameter measured at baseline was influenced by treatment with 1,25-dihydroxyvitamin $D_{3}$, either i.v. or orally.

Figure 1 presents plasma concentrations of glucose and insulin during OGTT at inclusion, $2 \mathrm{~h}$ after i. v. 1,25-dihydroxyvitamin $D_{3}$ and after 2 weeks of daily oral 1,25-dihydroxyvitamin $\mathrm{D}_{3}$. There was no statistically significant difference between the curves regarding glucose levels, whereas the levels of insulin

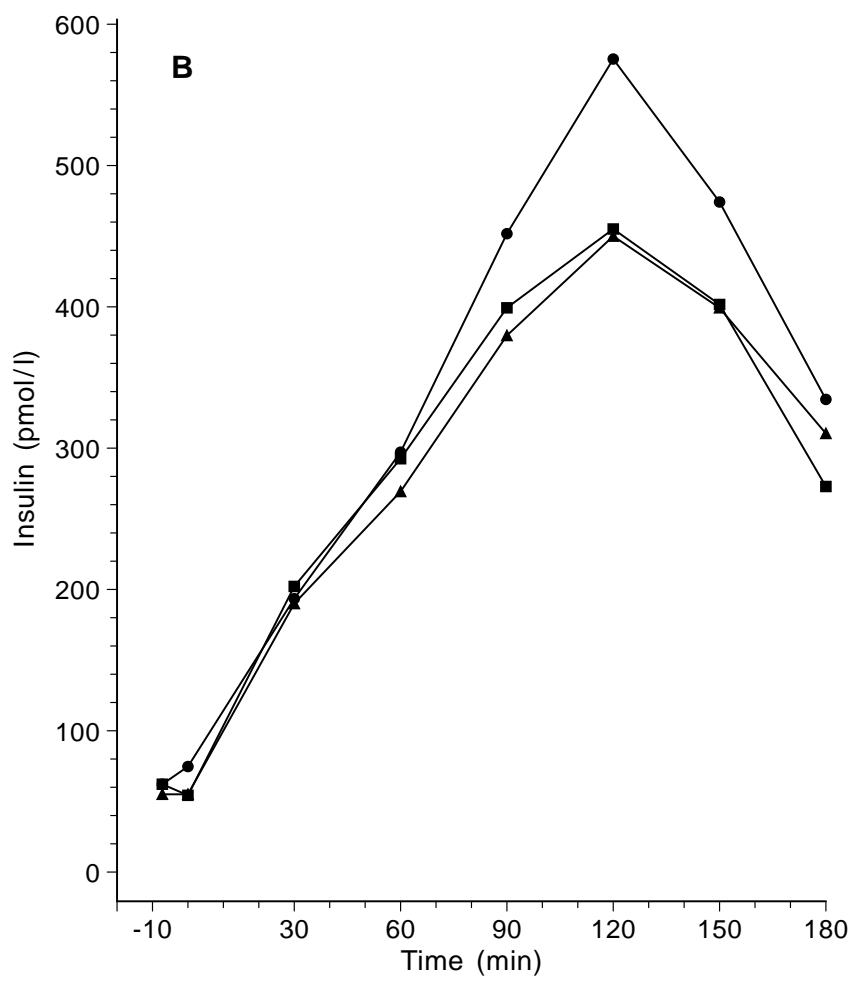

tended to be lower at 120 min after both i. v. and oral treatment. Calculating insulin changes in percent of baseline values the difference became statistically significant comparing before and after i.v. 1,25-dihydroxyvitamin $\mathrm{D}_{3}(645 \pm 296$ vs $543 \pm 234 \%, p<0.05$, mean $\pm S D)$, whereas the difference between the mean values before and after oral 1,25-dihydroxyvitamin $D_{3}$ did not reach statistical significance ( $645 \pm 296$ vs $588 \pm 293 \%, p=0.13$ ) (Table 2 ).

There was a significant negative relationship between the concentrations of glucose and 1,25-dihydroxyvitamin $D_{3}(r=-0.41, p<0.01)$ and a positive relationship between concentrations of glucose and parathyroid hormone $(r=0.31, p=0.07)$, when all measurements were included in the analysis. The concentrations of glucose correlated significantly with insulin $(r=0.51, p<0.002)$. In a multiple regression analysis including concentrations of 1,25-dihydroxyvitamin $D_{3}$, insulin, parathyroid hormone, weight and height as independent parameters and glucose as dependent, only 1,25-dihydroxyvitamin $\mathrm{D}_{3}$ and insulin turned out to be included in the final model $\left(r^{2}=0.73, p<0.0001\right)$.

\section{Discussion}

There are several reports on the beneficial effects of 1,25-dihydroxyvitamin D on glucose tolerance. H owever, the dose given and the administration route differ markedly between the studies. In vitamin $D$ depleted humans supplements of $2000 \mathrm{IU}$ vitamin $\mathrm{D}_{3}$ per day [7] or a single i.m. dose of $100000 \mathrm{IU}$ were 
given [8]. N on-insulin-dependent diabetic subjects received orally $2 \mu \mathrm{g} 1 \alpha(\mathrm{OH}) \mathrm{D}_{3}$ [11]. In renal failure other regimes were used $[13,14]$. These differences make any comparison of the studies difficult. Furthermore, it is difficult to select the optimal treatment regimen.

In the present study, we evaluated the effect of i.v. 1,25-dihydroxyvitamin $D_{3}$ using a regimen previously described [13]. The pregnant women received a lower oral dose than that used by others $[11,14]$ to avoid hypercalcaemia in pregnancy.

O ur study shows that i.v. 1,25-dihydroxyvitamin $\mathrm{D}_{3}$ significantly decreased fasting glucose level in GD M. H owever, this effect was short-lived since we could not demonstrate any effect on plasma glucose levels during the following O G TT. N either could daily oral supplementation of Etalpha prescribed for 2 weeks after the initial i. v. bolus of 1,25-dihydroxyvitamin $D_{3}$ prevent the initially observed effect on fasting glucose. In contrast the level of insulin apparently decreased after i.v. and oral treatment.

The explanation for the lower glucose level prior to OG TT after i. v. injection of 1,25-dihydroxyvitamin $\mathrm{D}_{3}$ in our study could not be attributed to an increased insulin level. This is in contrast to most other studies suggesting that the effect of 1,25-dihydroxyvitamin $D$ on glucose metabolism may be through influence on insulin secretion from the pancreatic beta cells $[1,2,8,11,13,23]$. However, in these studies the results are difficult to interpret, since they either report no or minimal changes in glucose or insulin levels $[11,13]$ or that both variables increased during vitamin $\mathrm{D}$ treatment [8].

A nother mode of action of 1,25-dihydroxyvitamin $\mathrm{D}_{3}$ on glucose metabolism could be increasing the cellular absorption of glucose, either directly or by enhancing the effect of insulin. In support of this hypothesis is our observation of lower levels of insulin during the OGTT after i.v. and oral treatment and no change in the level of glucose. The presence of a positive relationship between the concentrations of glucose and 1,25-dihydroxyvitamin $D_{3}$ further substantiates this hypothesis. A Iso, 1,25-dihydroxyvitamin $D_{3}$ was included in the regression analysis explaining $73 \%$ of the variation in fasting plasma glucose together with insulin. However, precise knowledge of the mechanism by which 1,25-dihydroxyvitamin $D_{3}$ interferes with glucose metabolism must await further studies.

We did not observe any influence of oral 1,25-dihydroxyvitamin $\mathrm{D}_{3}$ on fasting levels of glucose and 1,25dihydroxyvitamin $D_{3}$. Since all patients had to return the remaining $E$ talpha tablets after the end of the study, we do not think that the low level of 1,25-dihydroxyvitamin $D_{3}$ was due to poor compliance, rather it may have been due to our use of a low dose $(0.25 \mu \mathrm{g} /$ day) compared to other studies using either $2 \mu \mathrm{g}$ or $0.5 \mu \mathrm{g}$ per day $[11,14]$. O $\mathrm{n}$ the other hand, this regimen decreased insulin levels to the same extent as i. v. administration. Since the patients received a restricted diet during the oral treatment study we cannot exclude that the change in diet may, in part, be responsible for the observed change in insulin levels.

We expected only minor changes in calcium homeostasis following i.v. 1,25-dihydroxyvitamin $D_{3}$ taking the short duration from injection and blood sampling into account. On the other hand, we expected that oral 1,25-dihydroxyvitamin $D_{3}$ would increase the intestinal calcium absorption. Since we used a rather low oral dose, this is probably the major reason for the lack of change in calcium-related parameters.

In conclusion, our results demonstrate that administration of 1,25-dihydroxyvitamin $D_{3}$ tends to decrease fasting glucose concentration in GDM. This may be due to increased insulin sensitivity, according to our findings of lower levels of insulin during OGTT. However, the exact mechanism by which 1,25-dihydroxyvitamin $D_{3}$ acts on glucose metabolism is not yet clear.

A cknowledgements. $\mathrm{H}$ andelsgartner $\mathrm{O}$ ve Buhl O lesen og fru E dith B uhl O Isen's mindelegat.

\section{References}

1. R oth J, B onner-Weir S, Norman A W, O rci L (1982) Immunocytochemistry of vitamin D-dependent calcium binding protein in chick pancreas: exclusive localization in betacells. E ndocrinology 110: 2216-2218

2. Clark SA, Stumph WE, Sar M, D eL uca HF, Tannaka Y (1980) Target cells for 1,25-dihydroxyvitamin $D_{3}$ in the pancreas. Cell Tissue R es 209: 515-520

3. Frankel BJ, H eldt A M , Grodsky G M (1980) Vitamin D deficiency inhibits pancreatic secretion of insulin. Science 209: 823-825

4. Certow BS, Sivitz W, B aranetsky NG, Clark SA, Waite A, D eL uca H F (1983) Cellular mechanisms of insulin release: the effects of vitamin $D$ deficiency and repletion on rat insulin secretion. E ndocrinology 113: 1511-1518

5. N yomba B L, B ouillon R, M oor P (1984) I nfluence of vitamin $D$ status on insulin secretion and glucose tolerance in the rabbit. Endocrinology 115: 191-197

6. Cade C, Norman A W (1986) R apid normalization/stimulation by 1,25 -dihydroxyvitamin $D_{3}$ on insulin secretion and glucose tolerance in the vitamin $\mathrm{D}$-deficient rat. Endocrinology 120: 1490-1499

7. G edik O, A Ikalin S (1986) E ffects of vitamin D deficiency and repletion on insulin and glucagon secretion in man. Diabetologia 29: 142-145

8. B oucher BJ, Mannan N, Noonan K, Hales CN, Evans SJ (1995) Glucose intolerance and impairment of insulin secretion in relation to vitamin $D$ deficiency in east $L$ ondon A sians. D iabetologia 38: 1239-1245

9. Storm TL, Sørensen O H , L und B , L und B , Christiansen J S, A ndersen A R, L umholtz IB , Parving HH (1983) V itamin $D$ metabolism in insulin-dependent diabetes mellitus. $M$ etab B one D is R elat R es 5: 107-110

10. B aumgartl HJ, Standl E, Schmidt-G ayk H, Kolb HJ , Janka HU, Ziegler A G (1991) Changes of vitamin $D_{3}$ serum 
concentrations at the onset of immune mediated type 1 (insulin dependent) diabetes mellitus. D iab R es 16: 145-148

11. Inomata S, Kadowaki S, Y amatani T, Fukase M, Fujita T (1986) E ffect of $1 \alpha(\mathrm{OH})$-vitamin $\mathrm{D}_{3}$ on insulin secretion in diabetes mellitus. B one and M ineral 1: 187-192

12. O rwoll E, R iddle M, Prince M (1994) E ffects of vitamin D on insulin and glucagon secretion in non insulin dependent diabetes mellitus. A m J Clin N utr 59: 1083-1087

13. M ak R HK (1992) Intravenous 1,25 dihydrocholecalciferol corrects glucose intolerance in haemodialysis patients. K idney Int 41: 1049-1054

14. Q uesada J M , M artin-M alo A, Santiago J et al. (1990) E ffect of calcitriol on insulin secretion in uremia. Nephrol D ial Transplant 5: 1013-1017

15. M ak R HK, DeFronzo R A (1992) Glucose and insulin metabolism in uremia. Nephron 61: 377-382

16. Kühl C (1975) Glucose metabolism during and after pregnancy in normal and gestational diabetic women. A cta E ndocrinol (Copenh) 79: 709-719

17. Frølich A, R udnicki M, Storm T, R asmussen N, H egedüs $L$ (1992) Impaired 1,25-dihydroxyvitamin D production in pregnancy-induced hypertension. E ur J O bstet Gynecol Reprod Bio 147: 25-29
18. Kouppala T (1988) A Iterations in vitamin D metabolites and minerals in diabetic pregnancy. G ynecol O bstet Invest 25: $99-105$

19. M imouni F, T sang R C, H ertzberg V S, N eumann V, E llis K (1989) Parathyroid hormone and calcitriol changes in normal and insulin dependent diabetic pregnancies. 0 bstet G ynecol 74: 49-53

20. A ndersen L, D inesen B, J ørgensen PN, Poulsen F, Røder ME (1993) E nzyme immunoassay for intact human insulin in serum or plasma. Clin Chem 39: $578-582$

21. I qbal SJ (1994) Vitamin D metabolism and the clinical aspects of measuring metabolites. A nn Clin Biochem 31: 109-124

22. N ussbaum SR, Zahradnik RJ, Lavigne JR et al. (1987) $\mathrm{H}$ ighly sensitive two-site immuno-radiometric assay of parathyrin, and its clinical utility in evaluating patients with hypercalcemia. Clin Chem 33: 1364-1367

23. Pike J W (1981) R eceptors for 1,25-dihydroxyvitamin $D_{3}$ in chick pancreas: a partial physical and functional characterization. J Steroid B iochem 16: 385-395

24. R eichel H, Koeffler H P, Norman A W (1989) The role of the vitamin $\mathrm{D}$ endocrine system in health and disease. $\mathrm{N}$ Engl J Med 32: 980-991 